\title{
Die ökologische Bedeutung der Spinnen in Forst-Ökosystemen, eine Literaturzusammenstellung
}

\section{Journal Article}

Author(s):

Nyffeler, Martin

Publication date:

1982-09

Permanent link:

https://doi.org/10.3929/ethz-a-005800894

Rights / license:

In Copyright - Non-Commercial Use Permitted

Originally published in:

Anzeiger für Schädlingskunde, Pflanzenschutz, Umweltschutz 55(9), https://doi.org/10.1007/BF01902703 
Anz. Schädlingskde., Pflanzenschutz, Umweltschutz 55, 134-137 (1982)

(C) 1982, Verlag Paul Parey, Berlin und Hamburg

ISSN 0340—7330/InterCode: ASUMDT

Entomologisches Institut der ETH, Zürich, Schweiz

\title{
Die ökologische Bedeutung der Spinnen in Forst-Ökosystemen, eine Literaturzusammenstellung ${ }^{1}$
}

\author{
Von M. NyFFELER
}

\begin{abstract}
The ecological importance

of spiders in forest ecosystems, a literature review

The most important literature on the ecological importance of spiders for forest ecosystems was compiled and analyzed. Forest spiders normally live in densities of 50 to 200 individuals $/ \mathrm{m}^{2}$ and prey, at least in Europe, predominantly on small soft-bodied insects (Diptera and Collembola mainly). However, bigger insects, including various forest pests, are also found in their prey. According to the present stand of knowledge the spiders of the soil surface seem to be important insect predators, whereas the ecological importance of spiders living on the trees is still controversial.
\end{abstract}

Spinnen gehören in Forst-Ơkosystemen zu den häufigsten Kleinräubern. So bestanden beispielsweise in Ostsachsen $42 \%$ der Fauna einer Kiefernkrone aus Spinnen (HöregotT, 1960). Von rund 840 mitteleuropäischen Spinnenarten bevorzugen nach VITÉ (1953) etwa 50\% den Wald. Die Besiedlungsdichten der Spinnen liegen in Wäldern in der Größenordnung zwischen 50 und 200 Individuen $/ \mathrm{m}^{2}$ (SCHMIDT, 1960; KirCHNER, 1964; Christophe u. Blandin, 1977, u. a.). In einzelnen Waldbiotopen waren sogar schon Spin-

1 Ausgeführt mit Unterstützung durch den Schweizerischen Nationalfonds zur Förderung der Wissenschaftlichen Forschung. nendichten von $>500$ Individuen $/ \mathrm{m}^{2}$ beobachtet worden (Pearse, 1946; Weidemann, 1978; Schaefer, 1980). Die Spinnendichte kann von Baumart zu Baumart stark variieren (KuUsisto, 1941). Auch die Artenzusammensetzung der Spinnenfauna unterscheidet sich manchmal wesentlich in Abhängigkeit der Baumart (STRATTON et al., 1979). In Wäldern werden Spinnen in verschiedenen Straten, von der Bodenoberfläche über die Kraut- und Strauchschicht bis hinauf in die Baumkronen gefunden. In den verschiedenen Straten haben sich zum Teil unterschiedliche Spinnenarten eingenischt (Albert, 1976; Uetz et al., 1978). Für europäische Wälder der gemäßigten und kälteren Zonen gilt folgendes: Im bodennahen Bereich herrschen oft kleine Netzspinnen aus den Familien Linyphiidae und Micryphantidae zahlenmäßig vor (HuHTA, 1965; Miller u. Obrtel, 1975; Christophe u. Blandin, 1977 , u. a.). Vor allem auf Böden lichter Wälder leben auch die netzlos jagenden Wolfspinnen (Familie Lycosidae). (Polenec, 1976; Nyffeler u. Benz, 1981 a). Diese erreichen zwar nicht so hohe Individuendichten wie die Netzspinnen, fallen dafür aber durch große Bewegungsaktivität auf. Im Kronenbereich der Nadelwälder stellen netzlos jagende Spinnen aus der $\mathrm{Fa}$ milie Thomisidae häufig den größten Individuenanteil, während in den Kronen der Laubwälder die Netzspinnenfamilien Linyphiidae, Micryphantidae 
und Theridiidae oft dominieren (AlberT, 1976). Für außereuropäische Wälder gelten $z$. T. andere faunistische Gesetzmäßigkeiten (vgl. Elliott, 1930; Gibson, 1947; Juillet, 1961; Gasdorf u. GoOdNight, 1963; Peck et al., 1971; Moulder u. Reichle, 1972).

Die meisten Spinnen sind zur Hauptsache Prädatoren von Insekten. Eine Ausnahme bildet die auch in Wäldern vorkommende Spinnengattung Ero (Familie Mimetidae), die sich vorwiegend von Spinnen ernährt (vgl. Рöтzsch, 1974). Eine kurze Beschreibung der Jagdstrategien häufiger Waldspinnen findet sich bei KirCHNER (1964). Auf der Bodenoberfläche ernähren sich viele Wolfspinnen und kleine Netzspinnen primär von Collembolen und anderen kleinen, weichhäutigen Arthropoden (EDGAR, 1969; HÅgVAR, 1973; NyfFeler u. BeNz, 1981 a, 1981 b). So wurde beispielsweise die bodennah lebende Wolfspinne Pardosa lugubris (Walck.) in einem Mischwald bei Zürich beim Aussaugen der Collembolen Orcbesella flavescens (Bourlet) ${ }^{2}$ und Tomocerus flavescens (Tullberg) ${ }^{2}$ beobachtet. Wolfspinnen fressen außer Insekten auch Arachniden; Kannibalismus kann für sie eine wichtige nahrungsökologische Bedeutung haben (EDGAR, 1969; Hagstrum, 1970; Hallander, 1970). Während von Wolfspinnen und kleinen Netzspinnen nur kleine, weichhäutige Insekten überwältigt werden können, werden von der auf europäischen Waldböden lebenden Trichterspinne Coelotes terrestris (Wider) häufig mittelgroße bis große Käfer getötet (TRETzel, 1961; Nentwig, 1981; Nyffeler u. Benz, 1981 b). Auf Waldböden spielt sich folglich Beuteselektion ab. Selektiver Beutefang scheint sich auch in der Vegetationsschicht zu ereignen (vgl. LuCzaK u. DabrowsKa-Prot, 1970). In der Vegetationsschicht stellen Fluginsekten (v. a. Dipteren) für viele Spinnenarten eine essentielle Nahrungsquelle dar (Turnbull, 1960; RuppertshoFEN, 1964; DabrowsKa-Prot u. LuCzaK, 1968; LuCzak u. Dabrowska-Prot, 1970; Thornhill, 1975; Uetz u. Biere, 1980; NyfFeler u. Benz, 1981 b). Nach europäischen Berichten handelt es sich dabei vor allem um kleine Insekten (NyFfELER u. BeNZ, 1981 b). Demgegenüber waren in Nordamerika sowohl mehrheitlich kleine als auch überwiegend mittelgroße Insekten fangende pflanzenbewohnende Radnetzspinnenarten beobachtet worden (UETZ et al., 1978; Uetz u. Biere, 1980).

Als polyphage Prädatoren töten Spinnen wirtschaftlich indifferente, nützliche und schädliche Insekten. In den Beutespektren von Spinnen sind schon zahlreiche Forstschädlinge nachgewiesen worden. Darunter befinden sich forstschädliche Käfer wie z. B. Maikäfer, Heldbock, Großer Brauner Rüsselkäfer, Gemeiner Graurüßler, Schwarzer Kiefernblattkäfer sowie mehrere Borkenkäferarten (KIRCHNER, 1964; RUPPERTShofen, 1964; Jennings u. Pase, 1975; Hiebsch u. Krause, 1976; Moor u. Nyffeler, 1982). Auch zahlreiche forstschädliche Schmetterlinge, wie beispielsweise Lärchenminiermotte, Eichenwickler, Fichtenwickler, Kiefernknospentriebwickler, Kiefernharzgallenwickler, Kiefernspanner, Buchenfrostspanner, Forleule, Nonne,

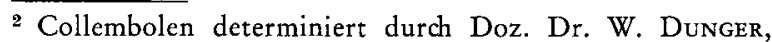
Staatliches Museum für Naturkunde, Görlitz, DDR.
Kiefernschwärmer und Weißer Bärenspinner konnten schon in der Beute von Spinnen festgestellt werden, wobei allerdings gewisse Netzspinnen wenig effektive Prädatoren von Schmetterlingen sind (vgl. unten) (Loughton et al., 1963; KrrCHNER, 1964; Brauns, 1976). Wie groß ist nun der Einfluß, den die Waldspinnen auf Insektenpopulationen ausüben können?

Die Biomasse der Spinnen auf der Bodenoberfläche von Forst-Okosystemen liegt in der Größenordnung von 2-5 kg Frischgewicht/ha (VAN DER DRIFT, 1951; Reichle u. Crossley, 1967; Moulder u. Reichle, 1972; Miller u. Obrtel, 1975). Die von den Spinnen der Streuschicht verzehrte Insektenmenge lag in einem amerikanischen Tulpenbaum-Okosystem in der Größenordnung von $12 \mathrm{~kg}$ Frischgewicht/ha/Jahr (MoulDER u. REICHLE, 1972). Das Energiebudget einer Wolfspinnenpopulation wurde in einem britischen ForstOkosystem auf ca. $9 \mathrm{~kg}$ Frischgewicht getötete Beute/ ha, in einem kalifornischen Forst-Ơkosystem auf etwas über $3 \mathrm{~kg} / \mathrm{ha}$ berechnet (HAGSTRUM, 1970; EDGAR, 1971). Dabei ist zu bedenken, daß Wolfspinnen nur einen Teil der gesamten epigäischen Spinnenfauna dieser beiden Okosysteme ausmachen. Amerikanische Wissenschaftler kamen zu dem Resultat, daß dic epigäischen Spinnen eine große Bedeutung als Prädatoren der Streufauna von Wäldern haben können (Clarke u. Grant, 1968; Moulder u. Reichle, 1972; Manley et al., 1976). Desgleichen mißt der deutsche Ókologe WeidemanN (1978) den epigäischen Spinnen, zusammen mit den anderen Streuschicht-Prädatoren, eine wichtige Funktion als Insektenprädatoren zu.

Im Gegensatz dazu ist die ökologische Bedeutung der Spinnen der Vegetationsschicht von Forst-O'okosystemen noch umstritten (VITÉ, 1953; KIRCHNER, 1964). Einzelne Feldstudien hatten gezeigt, daß gewisse Spinnenarten in der Vegetation ineffektiv gegen Lepidopteren-Schädlinge waren (Pointing, 1966; Kirchner, 1967; Furuta, 1977). Nach Angaben dieser Autoren konnten einzelne Spinnenarten Schmetterlingspopulationen um höchstens $5 \%$ reduzieren. $\mathrm{Zu}$ einem etwas höheren Mortalitätswert gelangte ENGel (1942). Er schätzte, daß bei einer Kiefernspannerkalamität $12-23 \%$ der Spannerpopulation von Spinnen vertilgt wurden, stellte jedoch zugleich fest, daß von Spinnen wesentlich weniger Spanner getötet werden konnten als von Wanzen. Die Ineffektivität gewisser Spinnenarten als Schmetterlingsfeinde dürfte z. T. mit dem spezifischen Fluchtvermögen der Schmetterlinge zusammenhängen (KrRCHNER, 1967; Nyffeler u. Benz, 1981 c). Andere Autoren hatten vermutet, daß Spinnen in der Vegetation von Forst-OKosystemen wichtige Prädatoren von Lepidopteren, Blattläusen und Mücken sein können (SUBKLEW, 1939; Juillet, 1961; DabrowsKa-Prot et al., 1968 a, b; LuCzaK, 1968; Fox u. Griffith, 1976). Die uneinheitliche Bewertung der Spinnen als Insektenprädatoren könnte damit zusammenhängen, daß von Biotop zu Biotop und von Jahr zu Jahr andere Umweltkonstellationen für Räuber-Beute-Interaktionen herrschen. So schwankte in Japan die von Spinnen verursachte Mortalitätsrate der Kastaniengallwespe in normalen Jahren zwischen 7 und $20 \%$ und war in 
einem anderen Jahr wesentlich höher (NAKAMURA u. Nakamura, 1977). Zudem hängt die ökologische Beurteilung der Spinnen stark davon ab, welche Spinnenart als Prädator welcher Beuteart studiert wird.

KIRCHNER (1964) schätzte die Vertilgungsleistung der Spinnen sämtlicher Straten (Boden + Vegetation) eines deutschen Forst-Ökosystems auf $100 \mathrm{~kg}$ Frischgewicht Insekten/ha/Jahr. Zum Vergleich sei festgehalten, daß in der Vegetation von Brachland, welches in der Sukzession eine Vorstufe des Waldes darstellt, sogar noch mehr als $100 \mathrm{~kg}$ Frischgewicht Insekten/ ha/Jahr von Spinnen zerstört werden (Nyfreler, 1982). Demgegenüber werden in der Vegetationsschicht von Getreidefeldern höchstens $1-2 \mathrm{~kg}$ Frischgewicht Insekten/ha/Jahr von Spinnen getötet (NYFFELER u. BENZ, 1979.

Außer als insektivore Prädatoren erfüllen die Spinnen innerhalb von Forst-Ökosystemen auch noch andere Funktionen. Spinnen können Konkurrenten von größengleichen Kleinräubern sein und dienen diversen anderen Tiergruppen als Haupt- oder Nebennahrung (z. B. für Vögel). Ferner stellen Spinnennetze in Wäldern wichtige Nahrungsquellen für Kleptoparasiten dar (THORNHILL , 1975).

Bevor die ökologische Bedeutung der Waldspinnen in allen ihren Aspekten erfaßt werden kann, werden in Zukunft noch zahlreiche Freilandstudien in den verschiedenen Waldtypen durchgeführt werden müssen. Vor allem wird der Kronenraum der Wälder noch besser untersucht werden müssen.

\section{Zusammenfassung}

Die wichtigste Literatur über die ökologische Bedeutung der Spinnen für Forst-Okosysteme wurde kurz zusammengestellt. Daraus geht hervor, daß Spinnen in Wäldern in Dichten von 50-200 Individuen $/ \mathrm{m}^{2}$ leben, und sich, zumindest in Europa, hauptsächlich von kleinen, weichhäutigen Insekten ernähren (v. a. Dipteren und Collembolen). In der Beute der Spinnen finden sich aber auch größere und sehr große Insekten, darunter verschiedene Forstschädlinge. Nach dem derzeitigen Stand des Wissens scheinen die Spinnen der Bodenoberfläche widhtige Insektenprädatoren $\mathrm{zu}$ sein, während die ökologische Bedeutung der im Stammund Kronenraum lebenden Spinnen noch umstritten ist.

\section{Literaturverzeichnis}

Albert, R., 1976: Zusammensetzung und Vertikalverteilung der Spinnenfauna in Buchenwäldern des Solling. Faun.-ökol. Mitt. 5, 65-80.

Brauns, A., 1976: Taschenbuch der Waldinsekten. Band I. Systematik und Okologie. Stuttgart: G. Fischer, $443 \mathrm{pp}$

Christophe, T., and P. Blandin, 1977: The spider community in the litter of a coppiced chestnut woodland (Forêt de Montmorency, Val d'Oise, France). Bull. Brit. Aradnol. Soc. 4, 132-140.

Clarke, R. D., and P. R. Grant, 1967: An experimental study of the role of spiders as predators in a forest litter community. Part 1. Ecology 49, 1152-1154.

DabrowsKa-Prot, E., and J. LuczaK, 1968: Studies on the incidence of mosquitoes in the food of Tetragnatha montana Simon and its food activity in the natural habitat. Ekol. Pol. A 16, 843-853.

Dabrowska-Prot, E., J. Luczak, and K. Tarwid, 1968 a: The role of prey and predator density in the process of mosquito reduction by spiders in field experiments. Ekol. Pol. A 16, 1-81.

Dabrowska-Prot, E., J. Luczak, and K. Tarwid, 1968 b: The predation of spiders on forest mosquitoes in field experiments. J. Med. Ent. 5, 252-256.
EDGAR, W. D., 1969: Prey and predators of the wolf spider Lycosa lugubris. J. Zool. (London) 159, 405-411.

Edgar, W. D., 1971: Aspects of the ecological energetics of the wolf spider Pardosa (Lycosa) lugubris (Walckenaer). Oecologia (Berl.) 7, 136-154.

ElliotT, F. R., 1930: An ecological study on the spiders of the beech-maple forest. Ohio J. Sci. 30, 1-22.

Engel, H., 1942: Über die Populationsbewegung des Kiefernspanners (Bupalus piniarius L.) in verschiedenen Bestandestypen. Z. ang. Ent. 29, 116-163.

Fox, R. C., and K. H. Grifrith, 1976: Predation of pine cinaran aphids by spiders. J. Georgia Ent. Soc. 11, $241-243$.

FURUTA, K., 1977: Evaluation of spiders, Oxyopes sertatus and O. badius (Oxyopidae) as a mortality factor of gypsy moth, Lymantria dispar (Lepidoptera: Lymantriidae) and pine moth, Dendrolimus spectabilis (Lepidoptera: Lasiocampidae). Appl. Ent. Zool. 12, 313-324.

Gasdorf, E. C., and C. J. Goodnight, 1963: Studies on the ecology of soil arachnids. Ecology 44, 261-268.

Gibson, W. W., 1947: An ecological study of the spiders of a river terrace forest in western Tennessee. Ohio J. Sci. 47, 38-44.

Hagstrum, D. W., 1970: Ecological energetics of the spider Tarentula kochi (Araneae: Lycosidae). Ann. Ent. Soc. Am. 63, 1297-1304.

HÅgVar, S., 1973: Ecological studies on a winter-active spider, Bolyphantes index (Thorell) (Araneida, Linyphiidae). Norsk ent. Tidsskr. 20, 309-314.

Hallander, H., 1970: Prey, cannibalism and microhabitat selection in the wolf spiders Pardosa chelata (O. E. Müller) and $P$. pullata (Clerck). Oikos 21, 337-340.

Hrebsch, H., and R. Krause, 1976: Zur Verbreitung und Lebensweise von Atypus affinis Eidhwald, 1830, in der Sächsischen Schweiz (Araneae, Atypidae). Faun. Abh. Mus. Tierk. Dresden 6, 69-88.

HÖREGOTT, H., 1960: Untersuchungen über die qualitative und quantitative Zusammensetzung der Arthropodenfauna in den Kiefernkronen. Beitr. Ent. 10, 891-916.

Hunta, V., 1965: Ecology of spiders in the soil and litter of Finnish forests. Ann. Zool. Fenn. 2, 260-308.

Jennings, D. T., and H. A. PASE, 1975: Spiders preying on Ips bark beetles. Southwest. Naturalist 20, 225-229.

JUILLET, J. A., 1961: Observations on athropod predators of the European pine shoot moth, Rhyacionia buoliana (Schiff.) (Lepidoptera: Olethreutidae), in Ontario. Can. Ent. 93, 195-198.

Kirchner, W., 1964: Bisher Bekanntes über die forstliche Bedeutung der Spinnen - Versuch einer Literaturanalyse. Waldhygiene 5, 161-198.

KIRCHNER, W., 1967: In welchem Maße beeinflussen Spinnen die Mortalitätsrate des Eichenwicklers (Tortrix viridana L.)? Waldhygiene 7, 23-31.

Kuvsisto, P., 1941: Studien über die Okologie und Tagesrhythmik von Pbylloscopus trocbilus acredula (L.) mit besonderer Berücksichtigung der Brutbiologie. Acta Zool. Fenn. 31, 1-120.

Loughton, B. G., C. Derry, and A.S. West, 1963: Spiders and the spruce budworm. In Morris (ed.), The dynamics of epidemic spruce budworm populations. Mem. Ent. Soc. Canada 31, 249-268.

LuCZAK, J., 1968: An attempt at quantitative estimation of some factors on the predator-prey system, on the example of spiders and mosquitoes. Ekol. Pol. B 14, $343-350$.

Luczak, J., and E. Dabrowska-Prot, 1970: Preliminary observations on the food of the spider Theridion pictum (Walck.) and its predators. Bull. Brit. Arachnol. Soc. 1, 109-111.

MANley, G. V., J. W. Butcher, and M. ZABIK, 1976: DDT transfer and metabolism in a forest litter macroarthropod food chain. Pedobiologia (Jena) 16, 81-98.

Miller, F., and R. Obrtel, 1975: Soil surface spiders in a lowland forest. Acta Sci. Nat. Brno 9, 1-40.

Moor, H., and M. NYFFELER, 1982: Eine borkenkäferfressende Spinne, Troxochrus nasutus Schenkel (Araneae, Erigonidae). Faun.-ökol. Mitr. (im Druck). 
Moulder, B. C., and D. E. ReICHLE, 1972: Significance of spider predation in the energy dynamics of forestfloor arthropod communities. Ecol. Monogr. 42, 473-493.

Nakamura, M., and K. Nakamura, 1977: Population dynamics of the chestnut gall wasp, Dryocosmus kuriphilus Yasumatsu (Hymenoptera: Cynipidae). V. Estimation of the effect of predation by spiders on the mortality of imaginal wasps based on the precipitin test. Oecologia (Berl.) 27, 97-116.

NenTwig, W., 1981: Insekten, Spinnennetze und Netzspinnen. Doktorarbeit, Universität Marburg/Lahn. In: Marburger Ent. Publ. 1 (5), 1-139.

Nyffeler, M., 1982: Field studies on the ecological role of the spiders as insect predators in agroecosystems. D. Sc. thesis, Swiss Federal Institute of Technology, Zurich.

NyfFeler, M., and G. Benz, 1979: Zur ökologischen Bedeutung der Spinnen der Vegetationsschicht von Getreide- und Rapsfeldern bei Zürich (Schweiz). Z. ang. Ent. 87, 348-376.

NyfFeler, M., und G. Benz, 1981 a: Einige Beobadhtungen zur Nahrungsökologie der Wolfspinne Pardosa lugubris (Walck.) (Araneae, Lycosidae). Dtsch. Ent. Z., N. F. 28, $(\mathrm{IV}-\mathrm{V}), 297-300$

NyfFeler, M., und G. Benz, 1981 b: Freilanduntersuchungen zur Nahrungsökologie der Spinnen: Beobachtungen aus der Region Zürich. Anz. Schädlinkskde., Pflanzenschutz, Umweltschutz 54, 33-39.

Nyffeler, M., und G. Benz, 1981 c: Einige Beobachtungen über die Flucht von adulten Lepidopteren aus den Netzen orbiteler Spinnen. Anz. Schädlingskde., Pflanzenschutz, Umweltschutz 54, 113-114.

PeARSE, A., 1946: Observations on the microfauna of the Duke forest. Ecol. Monogr. 16, 127-150.

Peck, W. B., L. O. Warren, and I. L. Brown, 1971: Spider fauna of shortleaf and loblolly pines in Arkansas. J. Georgia Ent. Soc. 6, 87-94.

Pornting, P. J., 1966: A quantitative field study of predation by the sheet-web spider, Frontinella communis, on European pine shoot moth adults. Canad. J. Zool. 44, 265-273.

Polenec, A., 1976: Die aktivitätsdominanten Bodenspinnen der Wälder Sloveniens (Arachnida: Araneae). Ent. Germ. 3, 130-134.

Pötzsch, J., 1974: Die Stachelspinne Ero furcata. Wissenschaft und Fortschritt 24, 278-279.

Rerchle, D. E., and D. A. Crossley, 1967: Investigations on heterotrophic productivity in forest insect com- munities. In: Petrusewicz, K. (ed.). Secondary productivity of terrestrial ecosystems (principles and methods) II. pp. 563-587. Warsaw: Polish Academy of Sciences. Ruppertshofen, H., 1964: Úber den Einsatz von Deckenund Radnetzspinnen im Forstschutz. Waldhygiene 5, $147-153$.

Schatfer, M., 1980: Sukzession von Arthropoden in verbrannten Kiefernforsten. II. Spinnen (Araneida) und Weberknechte (Opilionida). Forstw. Centralblatt 99, $341-356$.

Schmidt, G., 1960: Spinnen im Haushalt unserer Wälder. Kosmos 56, 199-204.

Stratton, G. E., G. W. Uetz, and D. G. Dillerry, 1979: A comparison of the spiders of three coniferous tree species. J. Arachnol. 6, 219-226.

SuвкLEW, W., 1939: Untersuchungen über die Bevölkerungsbewegung des Kiefernspanners (Bupalus piniarius L.). Mitt. Forstwirtsch. u. Forstwissensch. 10, 10-51. Zit. VITÉ, J. P., 1953.

Thornhill, R., 1975: Scorpionflies as kleptoparasites of web-building spiders. Nature (Lond.) 258, 709-711.

Tretzel, E., 1961: Biologie, Okologie und Brutpflege von Coelotes terrestris (Wider) (Araneae, Agelenidae). Teil I: Biologie und Ókologie. Z. Morph. Okol. Tiere 49, 658 bis 745 .

Turnbull, A. L., 1960: The prey of the spider Linyphia triangularis (Clerck) (Araneae, Linyphiidae). Can. J. Zool. 38, 859-873.

UETZ, G. W., and J. M. BIERE, 1980: Prey of Micrathena gracilis (Walckenaer) (Araneae: Araneidae) in comparison with artificial webs and other trapping devices. Bull. Brit. Arachnol. Soc. 5, 101-107.

Uetz, G. W., A. D. Johnson, and D. W. Schemske, 1978: Web placement, web structure, and prey capture in orbweaving spiders. Bull. Brit. Arachnol. Soc. 4, 141-148.

VAN DER DRIFT, J., 1951: Analysis of the animal community in a beech forest floor. Tijdschr. Ent. 94, 1-168. VITÉ, J. P., 1953: Untersuchungen über die ökologische und forstliche Bedeutung der Spinnen im Walde. Z. ang. Ent. 34, 313-334.

Weidemann, G., 1978: Uber die Bedeutung von Insekten im Okosystem Laubwald. Mitt. dtsch. Ges. allg. angew. Ent. 1, 196-204.

Anschrift des Verfassers: Dipl. Ing.-Agr. Martin NyfFeLER, Entomologisches Institut, Eidgenössische Technische Hochschule, ETH-Zentrum, CH-8092 Zürich (Schweiz). 